\title{
ChaC glutathione specific $\gamma$-glutamylcyclotransferase 1 inhibits cell viability and increases the sensitivity of prostate cancer cells to docetaxel by inducing endoplasmic reticulum stress and ferroptosis
}

\author{
SAIFEI HE ${ }^{1 *}$, MIAO ZHANG $^{1 *}$, YING YE $^{1}$, JUHUA ZHUANG $^{2}$, XING MA $^{2}$, YANAN SONG ${ }^{1}$ and WEI XIA ${ }^{2}$ \\ ${ }^{1}$ Central Laboratory and ${ }^{2}$ Department of Nuclear Medicine, The Seventh People's Hospital of \\ Shanghai University of Traditional Chinese Medicine, Pudong, Shanghai 200137, P.R. China
}

Received May 9, 2020; Accepted February 23, 2021

DOI: $10.3892 /$ etm.2021.10429

\begin{abstract}
The present study aimed to determine the effects and mechanism of ChaC glutathione specific $\gamma$-glutamylcyclotransferase $1(\mathrm{CHAC} 1)$ on cell viability and the sensitivity of prostate cancer cells to docetaxel. Compared with non-tumor human prostate epithelial RWPE-1 cells, the mRNA and protein levels of CHAC1 significantly decreased in two prostate cancer cell lines, DU145 and 22RV1, as measured by quantitative polymerase chain reaction and western blot analysis $(\mathrm{P}<0.05)$. The cell viability and glutathione $(\mathrm{GSH})$ levels were significantly inhibited in prostate cancer cells following overexpression of CHAC1 $(\mathrm{P}<0.01)$, while they were significantly increased in DU145 cells transfected with CHAC1 siRNA $(\mathrm{P}<0.05)$, but not in 22RV1 cells $(\mathrm{P}>0.05)$. The expression levels of several endoplasmic reticulum (ER) stress-related factors were then measured by western blot analysis. Following transfection with plasmid overexpressing CHAC1, ER markers, BIP and CHOP levels, were significantly upregulated $(\mathrm{P}<0.01)$, while $\mathrm{GSH}$ co-treatment decreased this upregulation. In addition, $\mathrm{CHAC} 1$ protein levels were significantly upregulated in cells treated with a ferroptosis activator $(\mathrm{P}<0.05)$. A liperflo reagent was then used to
\end{abstract}

Correspondence to: Professor Wei Xia, Department of Nuclear Medicine, The Seventh People's Hospital of Shanghai University of Traditional Chinese Medicine, 358 Datong Road, Pudong, Shanghai 200137, P.R. China

E-mail: awingxia@163.com

Professor Yanan Song, Central Laboratory, The Seventh People's Hospital of Shanghai University of Traditional Chinese Medicine, 358 Datong Road, Pudong, Shanghai 200137, P.R. China

E-mail: synabc.123@163.com

${ }^{*}$ Contributed equally

Key words: ChaC glutathione specific $\gamma$-glutamylcyclotransferase 1, prostate cancer, endoplasmic reticulum stress, ferroptosis, cell viability, sensitivity to docetaxel determine intracellular lipid peroxide levels. The intracellular lipid peroxides levels were significantly increased following CHAC1-overexpression $(\mathrm{P}<0.05)$, while GPX4 protein levels were significantly decreased $(\mathrm{P}<0.01)$. The cell viability was significantly inhibited $(\mathrm{P}<0.001)$ even with $1 \mathrm{nM}$ docetaxel (DTX) and a plasmid overexpressing $\mathrm{CHAC1}$, while the effect of inhibition was not significant at $1 \mathrm{nM}$ of DTX alone $(\mathrm{P}>0.05)$. This inhibition was also eliminated following the addition of a ferroptosis inhibitor. In summary, CHAC1 may inhibit cell viability and increase the sensitivity of prostate cancer cells to DTX. The cellular mechanism may involve the induction of ER stress and ferroptosis. The results of the present study identified a potentially novel therapeutic target for prostate cancer, which may be useful in patients with castration-resistant prostate cancer.

\section{Introduction}

It was estimated that there were almost 1.3 million new worldwide cases of prostate cancer and 359,000 associated deaths in 2018, ranking prostate cancer as the second most common cancer and the fifth leading cause of cancer-associated mortality in males (1). Although androgen deprivation therapy (ADT) has been significant for the treatment of prostate cancer, numerous patients eventually become insensitive to the therapy and progress to incurable castration-resistant prostate cancer (CRPC) $(2,3)$. Therefore, identifying targets associated with prostate cancer occurrence and development is vital for the development of novel therapeutic targets.

ChaC glutathione specific $\gamma$-glutamylcyclotransferase 1 (CHAC1) was first identified in mammalian cells in 2009 as a new component of the unfolded protein response (UPR) pathway (4). It is induced in response to endoplasmic reticulum (ER) stress (5). CHAC1 is a proapoptotic ER stress protein downstream of the pancreatic EIF2 $\alpha$ kinase-ATF4 pathway and appears to be important for human physiology and disease (6). CHAC1 was observed to have $\gamma$-glutamyl cyclotransferase activity (7) and overexpression leads to a robust depletion of glutathione (GSH) (8). A previous study reported that GSH depletion may stimulate ferroptosis (9). Ferroptosis is a novel programmed cell death mechanism that is characterized by the 
accumulation of reactive oxygen species (ROS) resulting from iron accumulation and lipid peroxidation $(10,11)$. Considering that GSH is a major intracellular antioxidant, CHAC1 may have an important role in cellular oxidative homeostasis (6).

However, the role of $\mathrm{CHAC} 1$ in prostate cancer, particularly in CRPC, remains unclear. The present study found that overexpression of CHAC1 in two CRPC cell lines, DU145 and 22RV1, inhibited cell viability and increased the sensitivity to docetaxel (DTX). The underlying mechanisms were likely associated with the inductive effects of $\mathrm{CHACl}$ on ER stress and ferroptosis.

\section{Materials and methods}

Cell culture. The human prostate epithelial RWPE-1 cell line was cultured in complete keratinocyte serum-free medium containing basal K-SFM (Invitrogen; Thermo Fisher Scientific, Inc.), supplemented with $50 \mu \mathrm{g} / \mathrm{ml}$ bovine pituitary extract (BPE; Invitrogen; Thermo Fisher Scientific, Inc.), $5 \mathrm{ng} / \mathrm{ml}$ epidermal growth factor (EGF; R\&D Systems, Inc.) and $1 \%$ antibiotic/antimycotic mixture (PSF). Human prostate cancer DU145 cells were cultured in Dulbecco's modified Eagle's medium supplemented with $10 \%$ fetal bovine serum (FBS; Gibco; Thermo Fisher Scientific, Inc.). The human prostate cancer 22RV1 cell line was cultured in RPMI-1640 medium (Gibco; Thermo Fisher Scientific, Inc.), supplemented with $10 \%$ FBS. All cell lines were obtained from the Cell Bank of Type Culture Collection of the Chinese Academy of Sciences and incubated in a humidified atmosphere with $5 \%$ $\mathrm{CO}_{2}$ at $37^{\circ} \mathrm{C}$.

GSH ethyl ester treatment. A total of $5 \mathrm{mM}$ GSH ethyl ester (Sigma-Aldrich; Merck KGaA) was added to CHAC1-transfected DU145 and 22RV1 cells at $24 \mathrm{~h}$ after transfection and incubated at $37^{\circ} \mathrm{C}$ for an additional $24 \mathrm{~h}$.

Cell treatment. A total of $50 \mu \mathrm{M}$ Erastin (MedChemExpress) was added to DU145 and 22RV1 cells and incubated at $37^{\circ} \mathrm{C}$ for $24 \mathrm{~h}$. DTX was purchased from Sanofi-Aventis Pharmaceuticals. Ferrostatin was purchased from MedChemExpress. DU145 and 22RV1 cells were treated with various concentrations $(0,0.1,1,5,10,20,50$ and $100 \mathrm{nM})$ of DTX alone or combined with $1 \mu \mathrm{M}$ ferrostatin at $37^{\circ} \mathrm{C}$ for $48 \mathrm{~h}$. DTX and ferrostatin were added to CHAC1-transfected DU145 and 22RV1 cells at $24 \mathrm{~h}$ after transfection and incubated at $37^{\circ} \mathrm{C}$ for an additional $48 \mathrm{~h}$.

Construction of the plasmid overexpressing CHAC1. The pcDNA3-Flag-CHAC1 plasmid was constructed by inserting the cDNA fragment encoding human $\mathrm{CHAC} 1$ protein (NM_024111.6) into the pcDNA3 vector with a Flag tag at its $\mathrm{N}$-terminal in frame to generate the Flag-tagged $\mathrm{CHAC1}$ fusion protein. The DNA construct was confirmed by sanger sequencing (Shanghai Personalbio Technology Co., Ltd.).

Plasmid transfection. Plasmid transfection was performed at $75 \%$ cell density of DU145 or 22RV1. The Flag-tagged CHAC1-overexpressing plasmid and its control vector was transfected using the Lipofectamine 3000 transfection reagent (Thermo Fisher Scientific, Inc.), according to the manufacturer's protocol, and incubated in a humidified atmosphere with $5 \% \mathrm{CO}_{2}$ at $37^{\circ} \mathrm{C}$ for $48 \mathrm{~h}$. Following transfection for $48 \mathrm{~h}$, the cells were harvested for subsequent assays.

Small interfering RNA (siRNA) transfection. siRNA transfection was performed at $50 \%$ cell density of DU145 or 22RV1. The siRNA specific to CHAC1 (5'-AUCUUCAAGGAGCGU CACCAC-3'; cat. no. SR312343; OriGene Technologies, Inc.) and its negative control (5'-GUUAAAUAGCGAUAGGAA UUC-3'; cat. no. SR30002; OriGene Technologies, Inc.) were commercially purchased. Transfection of siRNA-CHAC1 and its control (final conc. $10 \mathrm{nM}$ ) was performed using the lipofectamine RNAiMAX reagent (Thermo Fisher Scientific, Inc.) according to the manufacturer's protocols and cells were incubated in a humidified atmosphere with $5 \% \mathrm{CO}_{2}$ at $37^{\circ} \mathrm{C}$ for $72 \mathrm{~h}$. Following transfection, the cells were harvested for subsequent assays at once.

RNA extraction and reverse transcription. Total cellular RNA was harvested using TRIzol reagent (Invitrogen; Thermo Fisher Scientific, Inc.), according to the manufacturer's protocols. RNA was then reverse transcribed to cDNA at $37^{\circ} \mathrm{C}$ for $15 \mathrm{~min}$ and $98^{\circ} \mathrm{C}$ for $5 \mathrm{~min}$ using the SuperScript First-Stand Synthesis system (Invitrogen; Thermo Fisher Scientific, Inc.).

Quantitative polymerase chain reaction ( $q P C R$ ). SYBR Green (Toyobo Life Science) qPCR was performed to determine the mRNA expression levels of $\mathrm{CHAC} 1$ and $\beta$-actin (used as an internal control). The following primers were used: CHAC1 forward, 5'-TGTGGATTTTCGGGTACGGC-3' and reverse, 5'-CTTGCTTACCTGCTCCCCTT-3'; and $\beta$-actin forward, 5'-GTTGCTATCCAGGCTGTGCTA-3' and reverse, 5'-TGTCACGCACGATTTCCCGCT-3'. qPCR was performed on ABI 7500 System (Applied Biosystems; Thermo Fisher Scientific Inc.) using the following thermocycling conditions: $95^{\circ} \mathrm{C}$ for $30 \mathrm{sec}$; followed by 40 cycles at $95^{\circ} \mathrm{C}$ for $5 \mathrm{sec} ; 60^{\circ} \mathrm{C}$ for $30 \mathrm{sec}$ and $72^{\circ} \mathrm{C}$ for $15 \mathrm{sec}$. mRNA expression levels of $\mathrm{CHAC} 1$ were normalized to $\beta$-actin mRNA expression levels. Relative CHAC1 mRNA levels were calculated using the comparative $2^{-\Delta \Delta C q}$ method (12).

Western blot analysis. Cells were harvested and centrifuged at $4^{\circ} \mathrm{C}, 2,000 \mathrm{x}$ g for $5 \mathrm{~min}$. Cells were then lysed in $1 \mathrm{X}$ sodium dodecyl sulfate (SDS) loading buffer (Beyotime Institute of Biotechnology). Protein concentrations of the lysates were measured using the BCA protein assay kit (Pierce; Thermo Fisher Scientific, Inc.). The lysates were boiled for $10 \mathrm{~min}$, cooled and then centrifuged at $4^{\circ} \mathrm{C}, 12,000 \mathrm{x} \mathrm{g}$ for $10 \mathrm{~min}$. A total of $30 \mu \mathrm{g} /$ lane protein extracts were loaded onto a $10 \%$ SDS-polyacrylamide gel and then electrophoresed and transferred onto a polyvinylidene fluoride membrane (EMD Millipore). The blots were blocked at room temperature for $1 \mathrm{~h}$ in skimmed milk in tris-buffered saline and incubated with primary antibodies overnight at $4^{\circ} \mathrm{C}$. Following washing, the blots were incubated with IR-dye based secondary antibodies (LI-COR) for $1 \mathrm{~h}$ at room temperature. Protein bands were visualized using an Odyssey scanner (LI-COR Biosciences). The densitometry of the protein bands was quantified using the Odyssey analyzer software (LI-COR Biosciences). Rabbit anti-CHAC1 (cat. no. HPA043505; dilution, 1:500; Atlas 

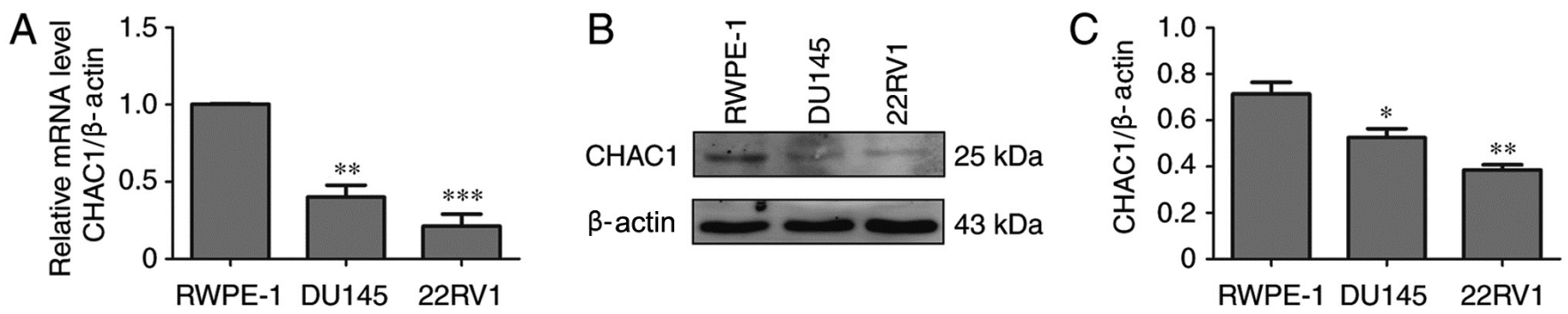

Figure 1. CHAC1 expression levels are reduced in prostate cancer cells compared with non-tumor prostate cells. (A) mRNA levels of CHAC1 in non-tumor human prostate epithelial RWPE-1 cells, and prostate cancer DU145 and 22RV1 cell lines were determined using reverse transcription-quantitative polymerase chain reaction. (B) The representative western blotting image of the expression of CHAC1 protein in RWPE-1, DU145 and 22RV1 cells. (C) Statistical data from (B) showing the expression level of CHAC1 protein compared with that of $\beta$-actin. $\beta$-actin was used as an internal control. Data are presented as the mean \pm standard error of the mean from three independent experiments. ${ }^{*} \mathrm{P}<0.05,{ }^{* *} \mathrm{P}<0.01,{ }^{* * *} \mathrm{P}<0.001$ vs. RWPE-1 cells. CHAC1, ChaC glutathione specific gamma-glutamylcyclotransferase 1 .

Antibodies AB), rabbit anti-BIP (cat. no. 3177; dilution, 1:500; Cell Signaling Technology, Inc.), mouse anti-CHOP (cat. no. 2895; dilution, 1:500; Cell Signaling Technology, Inc.), rabbit anti-LC3B (cat. no. 3868; dilution, 1:500; Cell Signaling Technology, Inc.), rabbit anti-GPX4 (cat. no. HPA058546; dilution, 1:500; Atlas Antibodies AB), rabbit anti-Flag (cat. no. 14793; dilution, 1:500; Cell Signaling Technology, Inc.), mouse anti- $\beta$-actin (cat. no. A5441; dilution, 1:500; Sigma-Aldrich; Merck KGaA) were used as primary antibodies and IR-dye based goat anti-mouse or goat anti-rabbit IgG (cat. nos. 925-68070/926-32211; dilution, 1:10,000; LI-COR Biosciences) were used as secondary antibodies.

Cell Counting kit-8 (CCK-8) assay. Cell viability was measured using the CCK-8 regent (Dojindo Molecular Technologies, Inc.), according to the manufacturer's protocols.

Glutathione (GSH) measurement. Cell GSH levels were measured using the glutathione assay kit (cat. no. CS0260; Sigma-Aldrich; Merck KGaA), according to the manufacturer's protocols.

Detection of intracellular lipid peroxides. DU145 or 22RV1 cells were seeded onto 96-well plates at a density of $1 \times 10^{4}$ cells/ well the day before transfection to perform image-based analysis for intracellular peroxides. After $16 \mathrm{~h}$, cells were transfected with Flag-tagged CHAC1-overexpressing plasmid or its control vector using the Lipofectamine $3000^{\circledR}$ reagent (Invitrogen; Thermo Fisher Scientific, Inc.) according to the manufacturer's protocols. A total of $48 \mathrm{~h}$ after transfection at $37^{\circ} \mathrm{C}$, intracellular lipid peroxides were detected using Hoechst 33342 (Beyotime Institute of Biotechnology) and Liperflo Reagent (Dojindo Molecular Technologies, Inc.), according to the manufacturer's protocols. Images were obtained using the Operetta high-content imaging system (PerkinElmer, Inc.) and analyzed using the Harmony v.4.8 software (PerkinElmer, Inc.) to determine intracellular lipid peroxide levels.

Statistical analysis. All experiments were performed at least in triplicate. Data are expressed as the mean \pm standard error of the mean. Statistical analysis was performed using a two-tailed unpaired t-test for two groups and one-way analysis of variance, followed by Turkey's test, for multiple groups using Prism5 software (GraphPad Software, Inc.).

\section{Results}

CHACl expression levels are decreased in prostate cancer cells. To determine the function of CHAC1 in prostate cancer, CHAC1 mRNA and protein levels were measured in non-tumor human prostate epithelial RWPE-1 cells and two prostate cancer cell lines, DU145 and 22RV1, by qPCR and western blotting. The results demonstrated that, compared with RWPE-1 cells, CHAC1 mRNA and protein levels were significantly decreased in DU145 and 22RV1 cells $(\mathrm{P}<0.05$; Fig. 1).

CHACl inhibits cell viability and decreases intracellular GSH levels. To determine the function of $\mathrm{CHAC} 1$ in prostate cancer, a CHAC1-overexpression plasmid and CHAC1 siRNA were constructed. Subsequently CHAC1 protein levels were determined in DU145 cells. CHAC1 was significantly upregulated in cells transfected with Flag-tagged CHACl-overexpressing plasmid, and downregulated in cells transfected with siRNA specific for CHAC1 (Fig. 2A).

The CCK-8 assay demonstrated that cell viability was significantly decreased in DU145 and 22RV1 cells transfected with $\mathrm{CHAC} 1$-overexpressing plasmid $(\mathrm{P}<0.01)$, while cell viability was significantly higher in DU145 cells transfected with CHAC1 siRNA $(\mathrm{P}<0.05)$, but not in 22RV1 cells $(\mathrm{P}>0.05$; Fig. 2B and C). It was also observed that GSH levels were significantly decreased in DU145 and 22RV1 cells transfected with $\mathrm{CHAC} 1$-overexpressing plasmid $(\mathrm{P}<0.01)$, while GSH levels were significantly increased in DU145 cells transfected with CHAC1 siRNA $(\mathrm{P}<0.05)$, but not in 22RV1 cells (Fig. 2D and E).

CHACl enhances ER stress. The present study subsequently focused on the associations between CHACl and ER stress in prostate cancer by measuring the expression levels of ER stress-related factors, BIP, CHOP and LC3B. As shown in Fig. 3A-E, BIP and CHOP levels were significantly upregulated in DU145 and 22RV1 cells following transfection with CHAC1-overexpressing plasmid $(\mathrm{P}<0.01)$, but LC3B levels were not $(\mathrm{P}<0.05)$. 

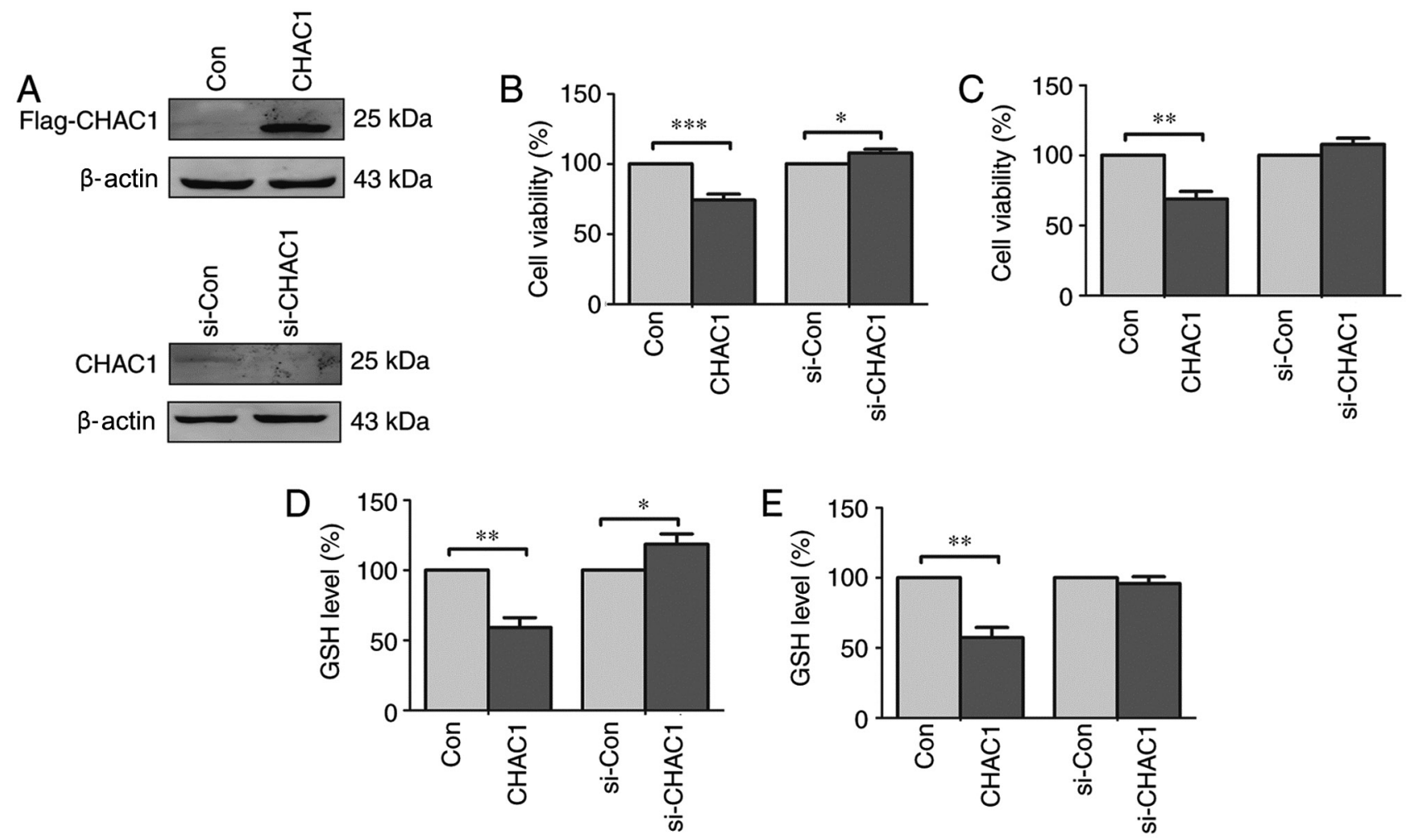

Figure 2. CHAC1 inhibits cell viability and reduces intracellular GSH levels in DU145 cells and 22RV1 cells. (A) The representative western blotting image of the expression of CHAC1 protein in DU145 cells. The top panel was the immunoblot confirmed by anti-Flag antibody in DU145 cells transfected with Flag-tagged CHAC1-overexpression plasmid (CHAC1) or with Con. The bottom panel was the immunoblot confirmed by anti-CHAC1 antibody in DU145 cells transfected with si-CHAC1 or with si-Con. (B) Cell viability of DU145 cells transfected with Flag-tagged CHAC1-overexpression plasmid (CHAC1) or with Con, or transfected with si-CHAC1 or with si-Con determined by CCK-8 assay. (C) Cell viability of 22RV1 cells transfected with Flag-tagged CHAC1-overexpression plasmid (CHAC1) or with negative plasmid (Con), or transfected with siRNA specific for CHAC1 (si-CHAC1) or with negative siRNA (si-Con) determined by CCK-8 assay. (D) Intracellular level of GSH in DU145 cells transfected with Flag-tagged CHAC1-overexpression plasmid (CHAC1) or with Con, or transfected with si-CHAC1 or with si-Con. (E) Intracellular level of GSH in 22RV1 cells transfected with Flag-tagged CHAC1-overexpression plasmid $(\mathrm{CHAC} 1)$ or with Con, or transfected with si-CHAC1 or with si-Con. Data are presented as the mean \pm standard error of the mean from three independent experiments. ${ }^{*} \mathrm{P}<0.05,{ }^{* *} \mathrm{P}<0.01,{ }^{* * *} \mathrm{P}<0.001$. CHAC1, ChaC glutathione specific gamma-glutamylcyclotransferase 1; Con, negative plasmid; si-CHAC1, CHAC1 small interfering RNA; si-Con, negative siRNA; CCK-8, Cell Counting kit-8; GSH, glutathione.

To further determine the association between CHAC1 and ER stress, DU145 and 22RV1 cells were transfected with CHAC1-overexpressing plasmid and exposed to GSH simultaneously. The results demonstrated that the increase in BIP and CHOP levels was decreased by exposure to GSH (Fig. 3F-I). This suggested that CHAC1 may induce BIP and CHOP expression by decreasing the levels of GSH to further enhance ER stress.

CHACl promotes ferroptosis. The role of CHAC1 in ferroptosis was subsequently investigated. It was observed that $\mathrm{CHAC} 1$ protein levels were significantly upregulated in DU145 and 22RV1 cells treated with Erastin, a type of ferroptosis activator $(\mathrm{P}<0.05$; Fig. $4 \mathrm{~A}$ and $\mathrm{B})$. Additionally, intracellular lipid peroxide levels were significantly increased following transfection with $\mathrm{CHAC} 1$-overexpressing plasmid $(\mathrm{P}<0.05$; Fig. 4C-E), while GPX4 protein levels were significantly decreased following CHAC1-overexpression $(\mathrm{P}<0.01$; Fig. 4F and G). These results indicated that $\mathrm{CHAC1}$ may induce ferroptosis.

CHACl increases the sensitivity of prostate cancer cells to DTX. Next, the effect of CHAC1 and ferroptosis on the sensitivity of prostate cancer cells to DTX was investigated.
The cell viability of DU145 and 22RV1 cells treated with different concentrations of DTX was measured. As shown in Fig. 5A and B, cell viability was only significantly inhibited when the concentration of DTX was $>5 \mathrm{nM}(\mathrm{P}<0.01)$. However, when prostate cancer cells were treated with $1 \mathrm{nM}$ DTX following transfection of the CHAC1-overexpressing plasmid, cell viability was significantly decreased $(\mathrm{P}<0.001$; Fig. 5C and D). Additionally, when prostate cancer cells were transfected with CHAC1-overexpressing plasmid followed by co-treatment with $1 \mathrm{nM}$ DTX and $1 \mu \mathrm{M}$ ferrostatin, a type of ferroptosis inhibitor, the effect of $\mathrm{CHACl}$ on decreasing cell viability was lessened. Cell viability was not significantly decreased by DTX ( $>0.05$; Fig. 5E and F). These results suggested that CHACl increases the sensitivity of prostate cancer cells to DTX by inducing ferroptosis.

\section{Discussion}

The present study demonstrated that $\mathrm{CHAC} 1$ expression levels in prostate cancer cells were significantly decreased, compared with normal prostate epithelial cells. CHAC1 expression levels were associated with cell viability and GSH levels. A previous study demonstrated that CHAC1 acts on cytoplasmic pools and its primary function was to alter the redox potential 
A

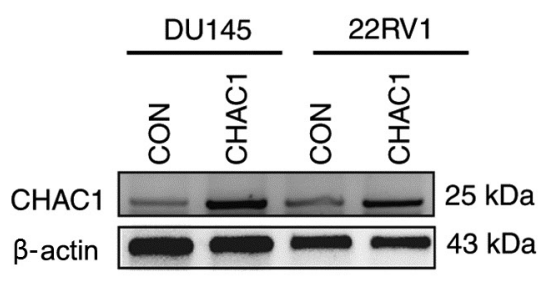

B

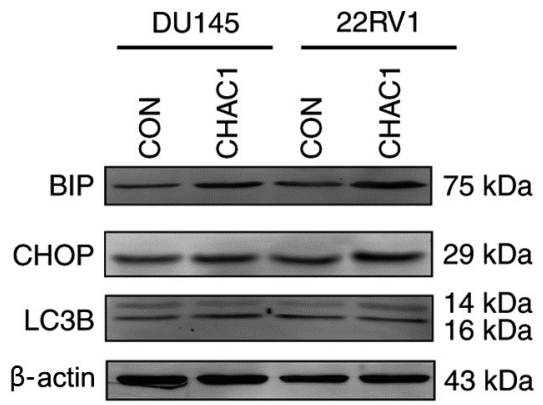

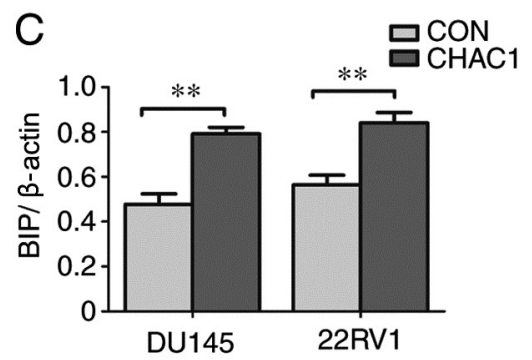

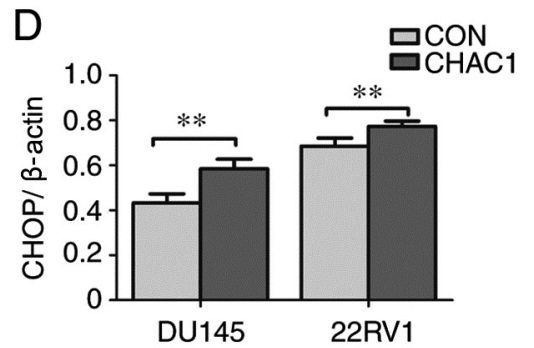

$\mathrm{E}$
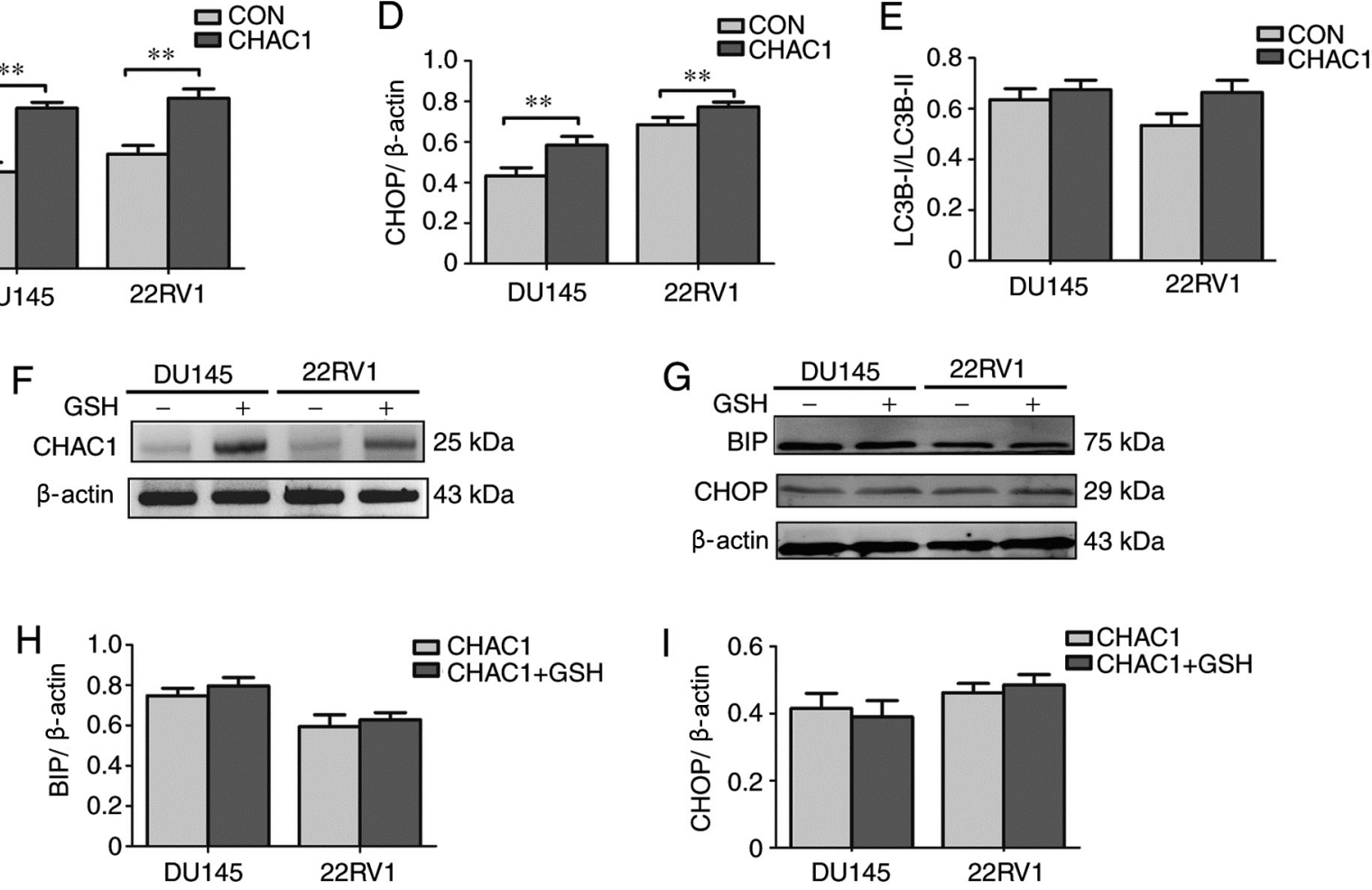

Figure 3. CHAC1 enhances ER stress in DU145 cells and 22RV1 cells. (A and B) The representative western blotting image of the expression of CHAC1, BIP, CHOP and LC3B protein in DU145 cells or 22RV1 cells following transfection with CHAC1-overexpression plasmid (CHAC1) or with Con. (C) Statistical data from (B) showing the expression level of BIP protein compared with that of $\beta$-actin. (D) Statistical data from (B) showing the expression level of CHOP protein compared with that of $\beta$-actin. (E) Statistical data from (B) showing the expression ratio of LC3B-I/LC3B-II. (F and G) The representative western blotting image of the expression of BIP and CHOP in CHAC1-overexpressing DU145 cells or CHAC1-overexpressing 22RV1 cells with (CHAC1 + GSH) or without (CHAC1) GSH replenishment by treatment with $5 \mathrm{mM}$ GSH ethyl ester. (H) Statistical data from (G) showing the expression level of BIP protein compared with that of $\beta$-actin. (I) Statistical data from $(\mathrm{G})$ showing the expression level of CHOP protein compared with that of $\beta$-actin. $\beta$-actin was used as an internal control. Data are presented as the mean \pm standard error of the mean from three independent experiments. ${ }^{* *} \mathrm{P}<0.01$. $\mathrm{CHACl}$, ChaC glutathione specific gamma-glutamylcyclotransferase 1; Con, negative plasmid.

that serves as activation signals (13). Results from this study and the previous study (13) suggested a possible role of CHAC1 in prostate cancer.

Cells may respond to an abrupt accumulation of secretory proteins within the ER through pathways, including UPR $(14,15)$. The state of cell health during ER stress ultimately decides cell fate (16). Previous studies have demonstrated that an increase in CHAC1 levels was associated with the activation of ER stress $(17,18)$. In the present study, overexpression of $\mathrm{CHAC} 1$ significantly upregulated the expression of ER stress-related factors, BIP and CHOP, while co-treatment with GSH inhibited ER stress. This indicated that $\mathrm{CHACl}$ may induce BIP and CHOP expression levels by decreasing GSH levels to further enhance
ER stress. However, in another previous study, CHAC1 siRNA treatment did not affect BIP and CHOP expression levels, while CHOP siRNA treatment inhibited CHAC1 expression (4). Therefore, the associations between $\mathrm{CHAC1}$ and CHOP may be more complex and require further investigation.

A previous study associated CHAC1 expression levels with ER stress induction (19), while the ER stress signaling pathway has been observed to contribute toward ferroptosis induction $(20,21)$. The increase in CHAC1 expression levels has been widely regarded as an indicator for early ferroptosis and have been associated with GSH degradation and the initiation of ferroptosis (22). This is consistent with the observations from the present study. 
A

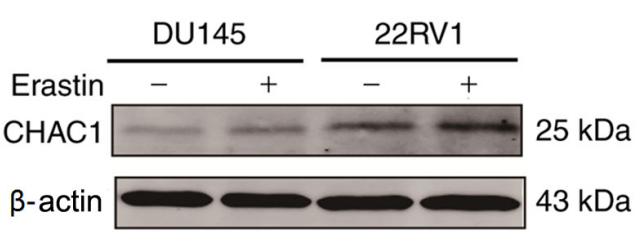

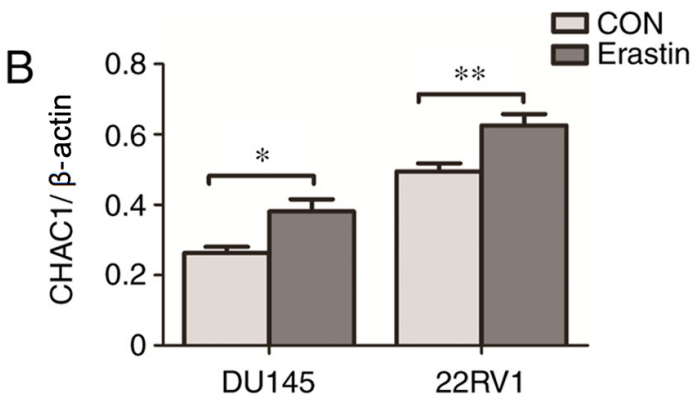

CHAC1
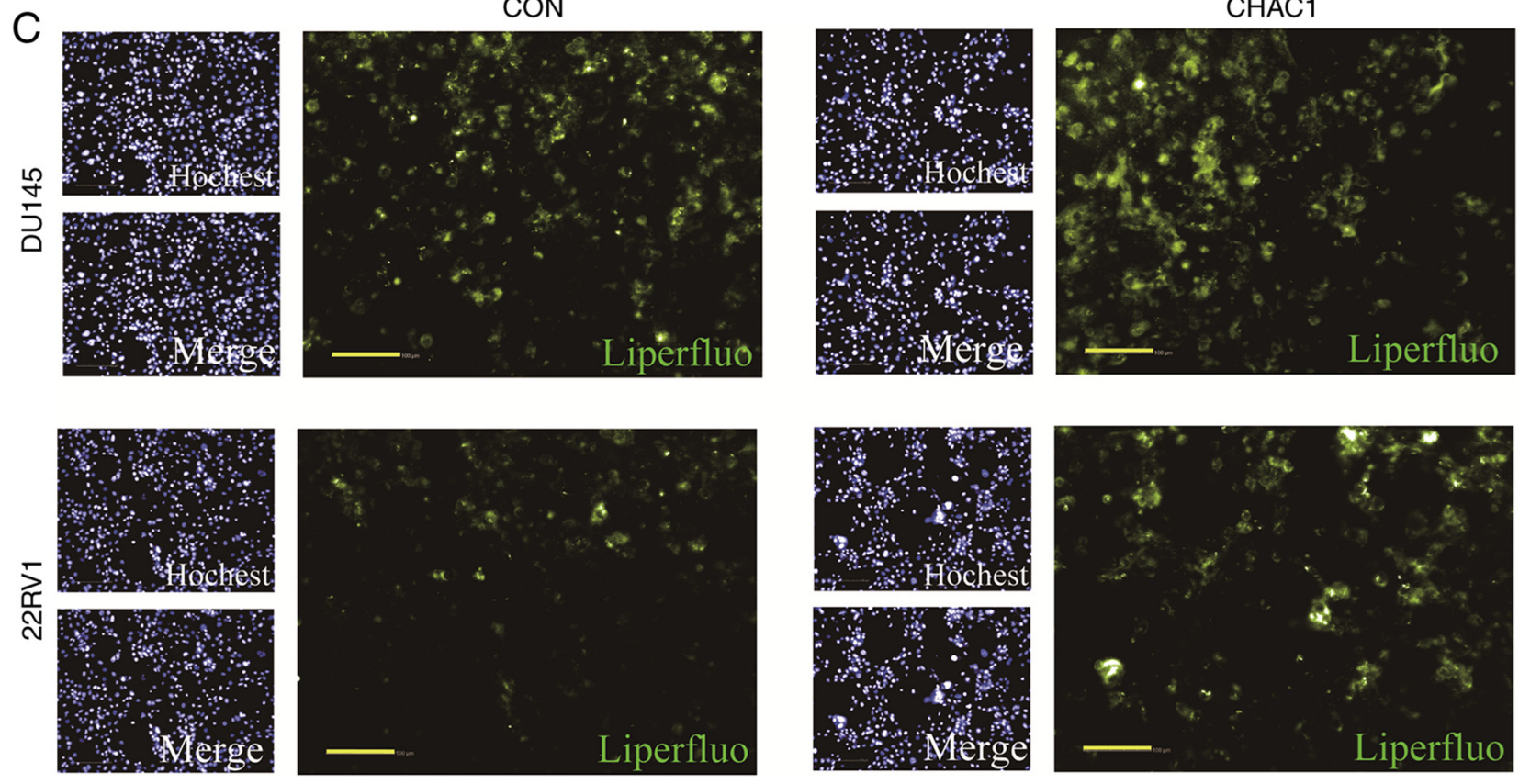

E
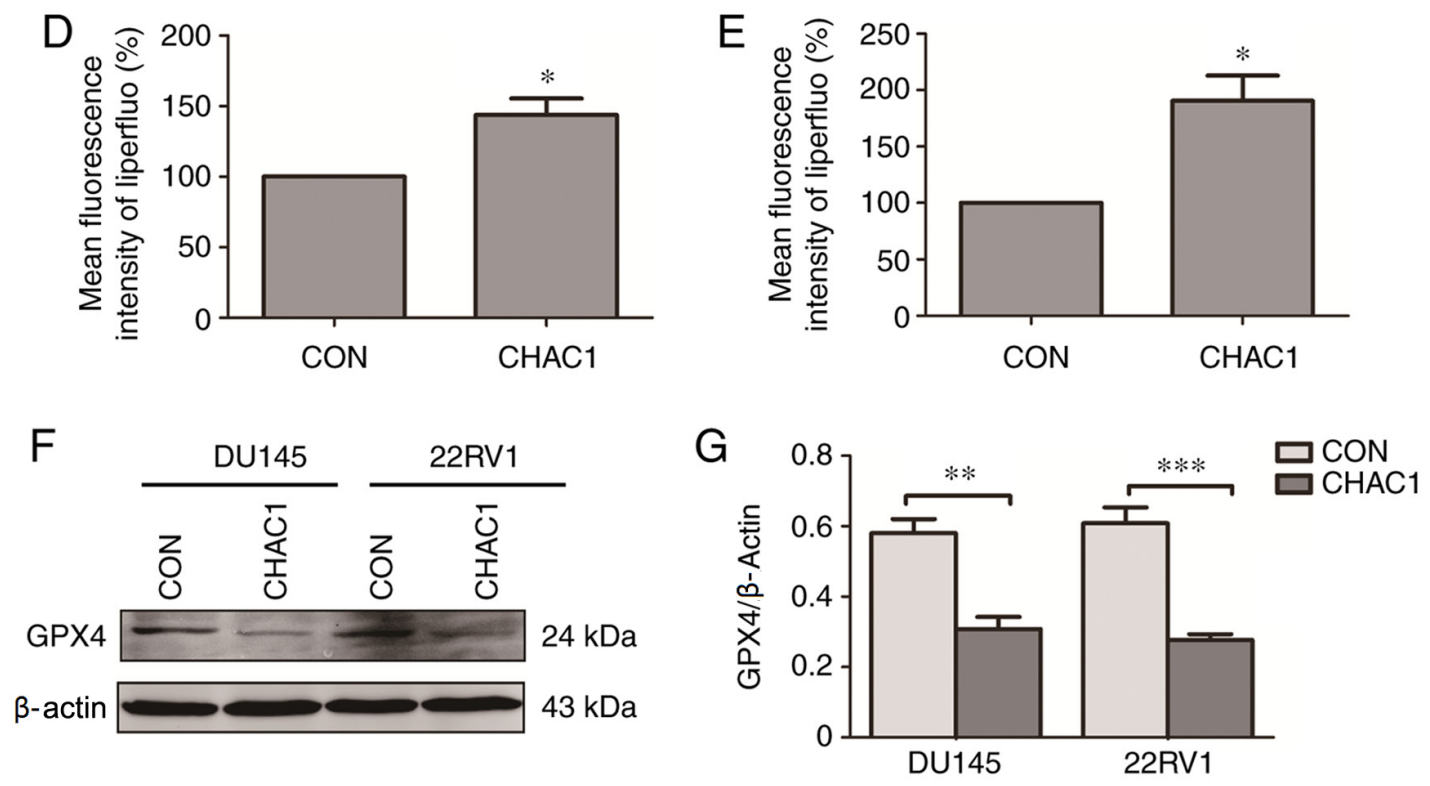

Figure 4. CHAC1 promotes ferroptosis in DU145 cells and 22RV1 cells. (A) Representative western blotting image of the expression of CHAC1 protein in DU145 cells or 22RV1 cells following treatment with (Erastin) or without (CON) $50 \mu \mathrm{M}$ erastin. (B) Statistical data from (A) showing the expression level of BIP protein compared with that of $\beta$-actin. (C) The representative high content screening image of intracellular lipid peroxides in DU145 and $22 \mathrm{RV} 1$ cells transfected with $\mathrm{CHACl}$-overexpression plasmid $(\mathrm{CHAC1})$ or with negative plasmid $(\mathrm{CON})$ detected using the LiperFluo probe. The nucleus was labeled using Hochest 33342 (Hochest). (D) Statistical data from (C) showing the intracellular lipid peroxides level by measuring mean fluorescence intensity of LiperFluo in DU145 cells. (E) Statistical data from (C) showing the intracellular lipid peroxides level by measuring mean fluorescence intensity of LiperFluo in 22RV1 cells. (F) The representative western blotting image of the expression of GPX4 protein in DU145 cells or 22RV1 cells following transfection with CHAC1-overexpression plasmid (CHAC1) or with Con. (G) Statistical data from (F) showing the expression level of GPX4 compared with that of $\beta$-actin. $\beta$-actin was used as an internal control. Data are presented as the mean \pm standard error of the mean from three independent experiments. ${ }^{*} \mathrm{P}<0.05,{ }^{* *} \mathrm{P}<0.01$, ${ }_{* * * *} \mathrm{P}<0.001$. CHAC1, ChaC glutathione specific gamma-glutamylcyclotransferase 1; Con, negative plasmid. 

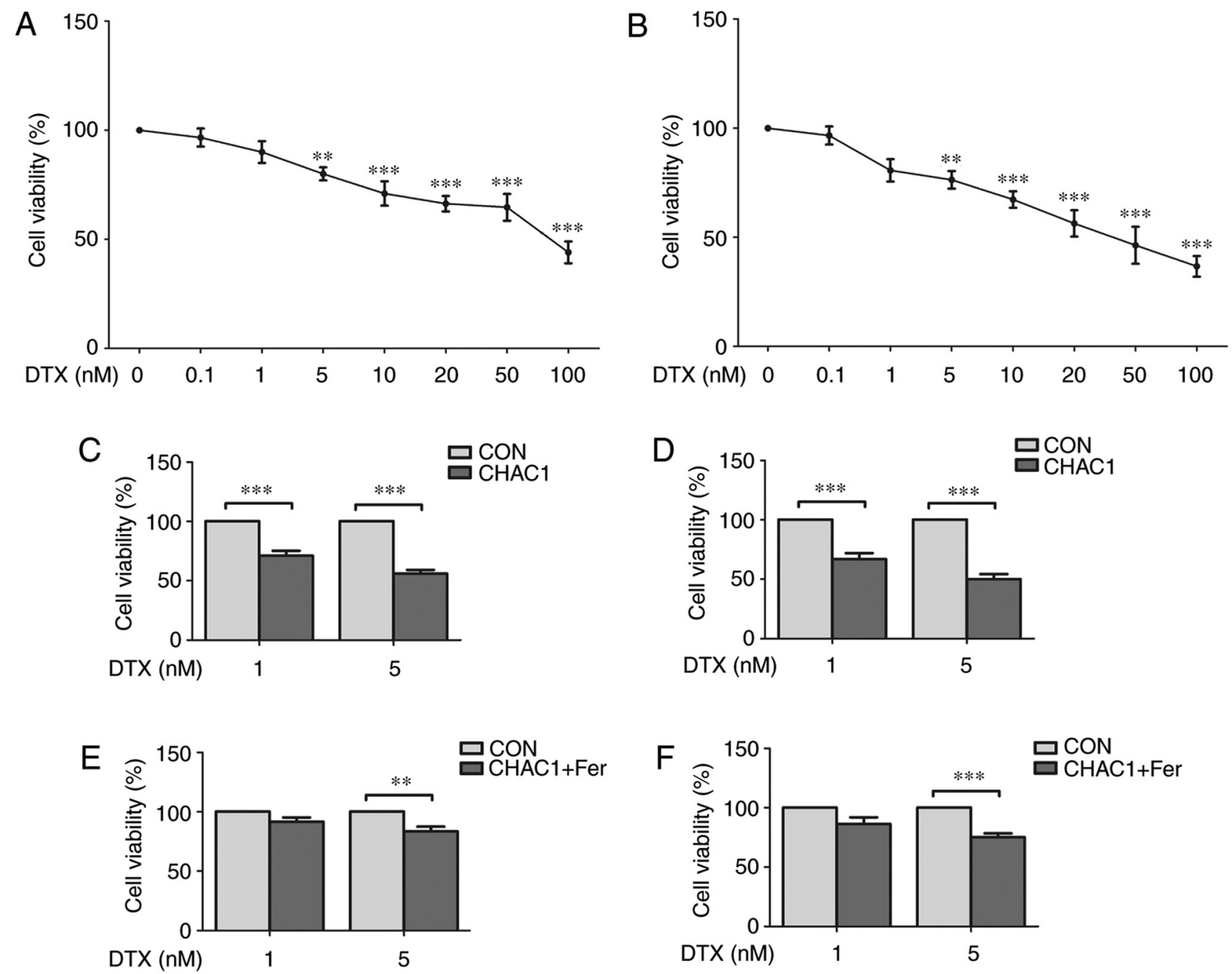

Figure 5. CHAC1 increases the sensitivity of prostate cancer cells to docetaxel. (A) Cell viability of DU145 cells treated with $0,0.1,1,5,10,20,50$ or $100 \mathrm{nM}$ DTX determined by CCK-8 assay. (B) Cell viability of 22RV1 cells treated with $0,0.1,1,5,10,20,50$ or $100 \mathrm{nM}$ DTX determined by CCK-8 assay. (C) Cell viability of DU145 cells treated with 1 or 5 nM DTX following transfection with CHAC1-overexpression plasmid (CHAC1) or with Con determined by CCK-8 assay. (D) Cell viability of 22RV1 cells treated with 1 or $5 \mathrm{nM}$ DTX following transfection with CHAC1-overexpression plasmid (CHAC1) or with Con determined by CCK-8 assay. (E) Cell viability of DU145 cells transfected with CHAC1-overexpression plasmid was determined by CCK-8 assay following co-treatment with $1 \mu \mathrm{M}$ Fer or DMSO (Con) and 1 or $5 \mathrm{nM}$ DTX. (F) Cell viability of 22RV1 cells transfected with CHAC1-overexpression plasmid was determined by CCK- 8 assay following co-treatment with $1 \mu \mathrm{M}$ Fer or DMSO (Con) and 1 or $5 \mathrm{nM}$ DTX. Data are presented as the mean \pm standard error of the mean from three independent experiments. ${ }^{* *} \mathrm{P}<0.01,{ }^{* * *} \mathrm{P}<0.001$. DTX, docetaxel; CHAC1, ChaC glutathione specific gamma-glutamylcyclotransferase 1; CCK-8, Cell Counting kit-8; Con, negative plasmid; Fer, ferrostatin.

Using prostate cancer cells, it was observed that overexpression of CHAC1 increased intracellular lipid peroxide levels and decreased GPX4 protein levels, resulting in the induction of ferroptosis. To the best of our knowledge, the present study was the first to demonstrate the effect of $\mathrm{CHACl}$ on ER stress and ferroptosis in prostate cancer cells. In addition, the ferroptosis process is defined by the iron-dependent accumulation of lipid reactive oxygen species and depletion of plasma membrane polyunsaturated fatty acids. Cancer cells with high level activities of the RAS-RAF-MEK or GCN2-eIF2 $\alpha$-ATF4 pathways may be sensitized to this process $(23,24)$. In future studies, it is worthy investigating the activation of those pathways.

CRPC therapy is not effective in decreasing mortality; therefore, the present study investigated whether CHAC1 may increase the sensitivity of prostate cancer cells to DTX by inducing ferroptosis. CHAC1 significantly increased the sensitivity of prostate cancer cells to DTX, and the effect was reversed following co-treatment with a ferroptosis inhibitor. This suggested an important role of CHAC1 in CRPC therapy. Combined therapy is gaining increased attention, and several studies have demonstrated synergistic effects (25-27). The results of the present study suggested that $\mathrm{CHAC1}$ may be a potential therapeutic target in combination with other therapeutics (including DTX) for the treatment of CRPC. Although the effects of CHAC1 have been confirmed in in vitro models, additional studies are required in the future to demonstrate its efficacy in other cell lines and in vivo. This should also be accompanied by clinical studies assessing the expression levels and activity levels of CHAC1.

In conclusion, it was found that $\mathrm{CHAC} 1$ could inhibit cell viability and increase the sensitivity of prostate cancer cells to DTX. The mechanism may involve the induction of ER stress 
and ferroptosis. The results of the present study may provide a potential novel therapeutic target for the treatment of prostate cancer, including CRPC. Additional studies are required to investigate the association between $\mathrm{CHAC} 1$ levels and the clinical outcome of patients with prostate cancer.

\section{Acknowledgements}

Not applicable.

\section{Funding}

The present study was supported by grants from National Natural Science Foundation of China (grant nos. 81904044 and 81904036), Shanghai Municipal Health Commission (grant no. 20174Y0044), Science and Technology Development Fund of Shanghai Pudong New Area (grant no. PKJ2017-Y14), Talents Training Program of Pudong Health Commission of Shanghai (grant no. PWRq2017-02) and Talents Training Program of Seventh People's Hospital of Shanghai University of Traditional Chinese Medicine (grant nos. XX2017-06 and XX2019-01).

\section{Availability of data and materials}

The datasets used and/or analyzed during the current study are available from the corresponding author on reasonable request.

\section{Authors' contributions}

WX and YS conceived the experiments. SH, MZ and XM conducted the experiments. YY and JZ analyzed the data. YS wrote the paper. WX and YS revised the paper. WX and YS confirmed the authenticity of all the raw data. All authors read and approved the final manuscript.

\section{Ethics approval and consent to participate}

Not applicable.

\section{Patient consent for publication}

Not applicable.

\section{Competing interests}

The authors declare that they have no competing interests.

\section{References}

1. Bray F, Ferlay J, Soerjomataram I, Siegel RL, Torre LA and Jemal A: Global cancer statistics 2018: GLOBOCAN estimates of incidence and mortality worldwide for 36 cancers in 185 countries. CA Cancer J Clin 68: 394-424, 2018.

2. Xiao L, Tien JC, Vo J, Tan M, Parolia A, Zhang Y, Wang L, Qiao Y, Shukla S, Wang X, et al: Epigenetic reprogramming with antisense oligonucleotides enhances the effectiveness of androgen receptor inhibition in castration-resistant prostate cancer. Cancer Res 78: 5731-5740, 2018.

3. Liao Y, Guo Z, Xia X, Liu Y, Huang C, Jiang L, Wang X, Liu J and Huang H: Inhibition of EGFR signaling with Spautin-1 represents a novel therapeutics for prostate cancer. J Exp Clin Cancer Res 38: 157, 2019.
4. Mungrue IN, Pagnon J, Kohannim O, Gargalovic PS and Lusis AJ: CHAC1/MGC4504 is a novel proapoptotic component of the unfolded protein response, downstream of the ATF4-ATF3-CHOP cascade. J Immunol 82: 466-476, 2009.

5. Perra L, Balloy V, Foussigniere T, Moissenet D, Petat H, Mungrue IN, Touqui L, Corvol $\mathrm{H}$, Chignard $\mathrm{M}$ and Guillot L: CHAC1 is differentially expressed in normal and cystic fibrosis bronchial epithelial cells and regulates the inflammatory response induced by pseudomonas aeruginosa. Front Immunol 9: 2823, 2018.

6. Crawford RR, Prescott ET, Sylvester CF, Higdon AN, Shan J, Kilberg MS and Mungrue IN: Human CHAC1 protein degrades glutathione, and mRNA induction is regulated by the transcription factors ATF4 and ATF3 and a bipartite ATF/CRE regulatory element. J Biol Chem 290: 15878-15891, 2015.

7. Kumar A, Tikoo S, Maity S, Sengupta S, Sengupta S, Kaur A and Bachhawat AK: Mammalian proapoptotic factor $\mathrm{ChaC} 1$ and its homologues function as $\gamma$-glutamyl cyclotransferases acting specifically on glutathione. EMBO Rep 13: 1095-1101, 2012.

8. Ogawa T, Wada Y, Takemura K, Board PG, Uchida K, Kitagaki K, Tamura T, Suzuki T, Tokairin Y, Nakajima Y and Eishi Y: CHAC1 overexpression in human gastric parietal cells with Helicobacter pylori infection in the secretory canaliculi. Helicobacter 24: e12598, 2019.

9. Yang WS, SriRamaratnam R, Welsch ME, Shimada K, Skouta R, Viswanathan VS, Cheah JH, Clemons PA, Shamji AF, Clish CB, et al: Regulation of ferroptotic cancer cell death by GPX4. Cell 156: 317-331, 2014.

10. Shi ZZ, Fan ZW, Chen YX, Xie XF, Jiang W, Wang WJ, Qiu YT and Bai J: Ferroptosis in carcinoma: Regulatory mechanisms and new method for cancer therapy. Onco Targets Ther 12: 11291-11304, 2019.

11. Bebber CM, Muller F, Prieto Clemente L, Weber J and von Karstedt S: Ferroptosis in cancer cell biology. Cancers (Basel) 12: 164, 2020.

12. Livak KJ and Schmittgen TD: Analysis of relative gene expression data using real-time quantitative PCR and the 2(-Delta Delta C(T)) method. Methods 25: 402-408, 2001.

13. Yadav S, Chawla B, Khursheed MA, Ramachandran R and Bachhawat AK: The glutathione degrading enzyme, Chac1, is required for calcium signaling in developing zebrafish: Redox as an upstream activator of calcium. Biochem J 476: 1857-1873, 2019.

14. Chadwick SR and Lajoie P: Endoplasmic reticulum stress coping mechanisms and lifespan regulation in health and diseases. Front Cell Dev Biol 7: 84, 2019.

15. Zeeshan HM, Lee GH, Kim HR and Chae HJ: Endoplasmic reticulum stress and associated ROS. Int J Mol Sci 17: 327, 2016.

16. Siwecka N, Rozpedek W, Pytel D, Wawrzynkiewicz A, Dziki A, Dziki Ł, Diehl JA and Majsterek I: Dual role of endoplasmic reticulum stress-mediated unfolded protein response signaling pathway in carcinogenesis. Int J Mol Sci 20: 4354, 2019.

17. Scheffer D, Kulcsár G, Nagyéri G, Kiss-Merki M, Rékási Z, Maloy $\mathrm{M}$ and Czömpöly T: Active mixture of serum-circulating small molecules selectively inhibits proliferation and triggers apoptosis in cancer cells via induction of ER stress. Cell Signal 65: 109426, 2020.

18. Cao SS and Kaufman RJ: Endoplasmic reticulum stress and oxidative stress in cell fate decision and human disease. Antioxid Redox Signal 21: 396-413, 2014.

19. Gagliardi M, Cotella D, Santoro C, Corà D, Barlev NA, Piacentini $\mathrm{M}$ and Corazzari M: Aldo-keto reductases protect metastatic melanoma from ER stress-independent ferroptosis. Cell Death Dis 10: 902, 2019.

20. Dixon SJ, Patel DN, Welsch M, Skouta R, Lee ED, Hayano M, Thomas AG, Gleason CE, Tatonetti NP, Slusher BS and Stockwell BR: Pharmacological inhibition of cystine-glutamate exchange induces endoplasmic reticulum stress and ferroptosis. Elife 3: e02523, 2014.

21. Lee YS, Lee DH, Choudry HA, Bartlett DL and Lee YJ: Ferroptosis-induced endoplasmic reticulum stress: Cross-talk between ferroptosis and apoptosis. Mol Cancer Res 16: 1073-1076, 2018. 
22. Dixon SJ, Lemberg KM, Lamprecht MR, Skouta R, Zaitsev EM, Gleason CE, Patel DN, Bauer AJ, Cantley AM, Yang WS, et al: Ferroptosis: An iron-dependent form of nonapoptotic cell death. Cell 149: 1060-1072, 2012.

23. Cao JY and Dixon SJ: Mechanisms of ferroptosis. Cell Mol Life Sci 73: 2195-2209, 2016.

24. Chen MS, Wang SF, Hsu CY, Yin PH, Yeh TS, Lee HC and Tseng LM: CHAC1 degradation of glutathione enhances cystine-starvation-induced necroptosis and ferroptosis in human triple negative breast cancer cells via the GCN2-eIF2 $\alpha$-ATF4 pathway. Oncotarget 8: 114588-114602, 2017.

25. Miao L, Guo S, Lin CM, Liu Q and Huang L: Nanoformulations for combination or cascade anticancer therapy. Adv Drug Deliv Rev 115: 3-22, 2017.
26. Xiao B, Ma L and Merlin D: Nanoparticle-mediated co-delivery of chemotherapeutic agent and siRNA for combination cancer therapy. Expert Opin Drug Deliv 14: 65-73, 2017.

27. Chen CK, Law WC, Aalinkeel R, Yu Y, Nair B, Wu J, Mahajan S, Reynolds JL, Li Y, Lai CK, et al: Biodegradable cationic polymeric nanocapsules for overcoming multidrug resistance and enabling drug-gene co-delivery to cancer cells. Nanoscale 6: $1567-1572,2014$

(1)(9) This work is licensed under a Creative Commons Attribution-NonCommercial-NoDerivatives 4.0 International (CC BY-NC-ND 4.0) License. 